\title{
Remodelling of subchondral bone in osteoarthritis: a histomorphometric study
}

\author{
G Amir, C J Pirie, S Rashad, P A Revell
}

\begin{abstract}
Aims: To determine whether remodelling of subchondral bone in osteoarthritis is related to anatomical and mechanical factors operating on the joint surface.

Methods: Ten femoral heads were examined. Patients due to have the femoral head removed because of osteoarthritis were given a double tetracycline label before surgery. The specimens were divided in three roughly equal parts, corresponding to the medial, central (weight bearing), and lateral aspects of the femoral heads. Undecalcified methylmethacrylate embedded sections were prepared. The subchondral bone was assessed for appositional bone formation by ultraviolet light microscopy, for resorptive activity by osteoclast count, and for trabecular bone volume by semiautomatic computerised image analysis. Appositional bone rate was also correlated with the presence or absence of overlying cartilage.
\end{abstract}

Results: Bone density was reduced in the medial aspect of the femoral heads but there was no significant difference between the appositional bone rate or the resorptive activity in the three areas. Nor was there any correlation between appositional bone rate in the subchondral bone and the presence or absence of overlying cartilage.

Conclusion: Remodelling of subchondral bone in osteoarthritis, at least in the short term, is fairly constant and is not related to weight bearing.

(f Clin Pathol 1992;45:990-992)

Osteoarthritis is becoming an increasingly important cause of morbidity as life expectancy increases. Its pathogenesis is unknown, partly because of difficulties in recognising early disease and obtaining human tissue for study at this stage. Therefore, only samples from advanced disease or bone from other parts of the skeleton can be studied for clues to its pathogenesis.

The damage to articular cartilage that is characteristic of osteoarthritis is accompanied by changes in the subchondral bone. It is not certain whether the bony changes precede or follow injury to the cartilage. Osteoarthritis may be a primary disorder of chondrocyte function with secondary changes in bone. Conversely, a defect in the subchondral bone, resulting in loss of its shock absorbing capacity, would transfer the stress of loading directly to the articular cartilage with secondary changes in the cartilage.

A great deal of work has been done to try and elucidate the physical and biochemical changes in articular cartilage. Minor degrees of fibrillation that occur with aging are seen as a normal physiological process. ${ }^{1}$ Biochemical changes in the non-calcified cartilage have been observed, but there is little evidence that these changes precipitate osteoarthritis. ${ }^{2-4}$

Changes in the subchondral bone may occur as a result of local factors, such as altered weight bearing, or as part of a generalised disease of bone such as Paget's disease. Simon et al induced cartilage damage in rabbits by repetitive excessive impulse loading. ${ }^{5}$ The damage was preceded by changes in the subchondral bone. Radin and Paul postulated that excessive impulse loading of the joint causes thickening of the subchondral bone as a result of repair of numerous microfractures. ${ }^{6}$ This bone is stiffer and less pliable so that the impact of loading is borne principally by the overlying cartilage, which, as a result, degenerates.

Several recent studies have indicated that the subchondral thickening seen in osteoarthritis may be part of generalised bone disease. An inverse correlation has been observed between osteoarthritis of the hip and osteoporosis. ${ }^{7-10}$ Increased cortical and trabecular bone mass has been shown in the peripheral skeleton of patients with osteoarthritis. ${ }^{11-15}$ Gevers suggests that this constitutionally thicker bone impairs the shock absorbing capacity in the subchondral region, therefore predisposing to osteoarthritis, and that these subjects are more likely to develop secondary osteoarthritis in the presence of local predisposing factors. ${ }^{15}$

The morphological appearances in osteoarthritis are well known; the subchondral bone undergoes a number of changes. Sclerosis occurs in areas of thinning and loss of the cartilage, particularly in the central area lateral to the fovea. Osteophyte production occurs around the circumference of the joint margin, and the so-called cysts, which contain fibrovascular tissue or metaplastic cartilage, are often surrounded by bone showing active new bone formation.

What initiates this subchondral remodelling? Wolff's law states that the structure of any bone is a function of the mechanical demands made on that bone. The load applied to any joint can be modified by many factors, such as weight, amount and type of exercise, occupation, 
integrity of neuromuscular control. Metabolic and systemic influences can also alter the subchondral bone, rendering it less able to withstand normal mechanical demands.

To evaluate bone remodelling in osteoarthritis, we measured appositional bone rate and osteoclast numbers in the subchondral bone in the femoral heads in cases of established disease, and compared the central high load bearing area with the medial and lateral areas, in an attempt to determine whether bone remodelling is related to anatomical and mechanical factors operating on the joint surface.

\section{Methods}

Ten femoral heads were examined. They came from five men and five women aged between 63 and 79 years. Before surgical removal of the femoral heads for osteoarthritis at joint replacement surgery, the patients were given a double tetracycline label $(250 \mathrm{mg}$ tetracycline four times a day for two periods of three days, separated by an interval of 11 days). The operation was performed two to three days after completion of the second dose. The surgical specimens were fixed in $70 \%$ methyl alcohol. A coronal slice through the middle of the femoral head and neck was taken in the plane of the fovea and this slice was divided into three roughly equal parts, corresponding to the medial, central, and lateral aspects of the femoral head. Undecalcified methylmethacrylate embedded sections were cut to a thickness of $10 \mu \mathrm{m}$ using a Jung $\mathrm{K}$ microtome. Slides were stained with haematoxylin and eosin, thionin, and von Kossa methods.

One section from each piece of bone was left unstained and mounted in Fluoromount for ultraviolet light microscopy. These sections were examined in a Zeiss fluorescence photomicroscope. Barrier filters BG38 and UG1 and excitor filters 50 and 65 were used and measurements were made at $\times 50$ magnification. The distance between tetracyclene labels was measured using a calibrated micrometer eyepiece.

Results were expressed as uncorrected values with respect to variations in the orientation of the double labelled osteoid seams, but the correction factor suggested by Frost ${ }^{1617}$

Appositional rate and osteoclast numbers in bone in medial, central, and lateral parts of osteoarthritic femoral head

\begin{tabular}{|c|c|c|c|}
\hline Case No & Medial & Central & Lateral \\
\hline \multicolumn{4}{|l|}{ Appositional rate ( $\mu \mathrm{m} /$ day) (uncorrected) } \\
\hline $\begin{array}{r}1 \\
2 \\
3 \\
4 \\
5 \\
6 \\
7 \\
8 \\
9 \\
10\end{array}$ & $\begin{array}{l}1.40(0.33) \\
1.46(0.58) \\
0.81(0.28) \\
2.05(1.01) \\
2.17(0.84) \\
1.86(0.64) \\
3.66(1.27) \\
1.77(0.82) \\
1.48(0.47) \\
1.23(0.30)\end{array}$ & $\begin{array}{l}1.05(0.29) \\
2.41(0.83) \\
1.07(0.29) \\
1.74(0.51) \\
1.56(0.44) \\
1.47(0.69) \\
3.39(1.40) \\
1.67(0.84) \\
1.63(0.44) \\
2.64(1.59)\end{array}$ & $\begin{array}{l}0.91(0.13) \\
2.81(1.48) \\
1.16(0.30) \\
1.57(0.30) \\
2.30(0.88) \\
1.24(0.37) \\
2.91(1.21) \\
1.46(0.36) \\
1.49(0.39) \\
2.13(1.35)\end{array}$ \\
\hline Mean & $1.79(0.77)$ & $1 \cdot 86(0 \cdot 74)$ & $1.80(0 \cdot 70)$ \\
\hline Corrected mean using Frost's formula'" & $1.33(0.57)$ & $1.33(0.55)$ & $1.33(0.52)$ \\
\hline $\begin{array}{l}\text { Osteoclast numbers (osteoclasts per } \mathrm{cm}^{2} \text { ): } \\
\text { Mean (SD) }\end{array}$ & $0.85(0.95)$ & $0.97(0.77)$ & $1.51(1.07)$ \\
\hline
\end{tabular}

was also calculated.

The numbers of the osteoclasts were assessed by defining an area in each region (medial, central, lateral) of each femoral head extending from the subchondral bone to the distal limit of the specimen. The total number of osteoclasts was counted in each area. Then the areas in which the counts were performed were measured using the Magiscan D image analysis system, calibrated so that real areas were measured. The results were expressed as osteoclasts per $\mathrm{cm}^{2}$.

Trabecular bone volume was measured by semiautomatic computerised image analysis using a Quantimet 720, examining 30 fields in each of the three regions of the femoral head.

\section{Results}

Appositional rate was measured at four equidistant points in 10 sites in each of the three parts of each femoral head. The appositional rate varied between 0.81 and $3.66 \mu \mathrm{m} /$ day (uncorrected) for the subchondral bone in the 30 separate sections of femoral heads examined. Details of the results for each of the three regions of the femoral head are shown in the table. There was no significant difference between the mean rates of appositional bone formation in the medial, central, and lateral parts of the femoral head. There were differences between regions in individual samples, but there was no specific pattern.

Haematoxylin and eosin stained sections adjacent to these examined by ultraviolet light microscopy were classified according to the nature of the articular surface related to (overlying) the bone without knowledge of the results obtained for appositional rate. The articular surface was formed by eburnated bone in 12 sections, cartilage in 10, and a combination of both in the remaining eight samples. Because loading through bone is different from that through cartilage, the appositional rates of the bone beneath eburnated and cartilaginous surfaces were compared. No significant difference was found (bone beneath eburnated surface 1.9 (SD 0.61) $\mu \mathrm{m} /$ day; bone beneath cartilage surface $1.54(0.59)$ $\mu \mathrm{m} /$ day).

Trabecular bone volume measured by computerised image analysis was $25 \cdot 2(7 \cdot 3) \%$ in the medial, $36.3(9 \cdot 1) \%$ in the central, and $32.0(8.7) \%$ in the lateral parts of the femoral heads. The medial femoral head was therefore of lower bone density than the other areas.

The subchondral bone was assigned to one of three categories (osteoporotic, intermediate (normal), osteosclerotic) on the basis of quantitative analysis. The mean appositional rate for osteoporotic areas of bone was $1.63(0.77)$ $\mu \mathrm{m} /$ day, for intermediate (normal) bone 1.82 $(0.63) \mu \mathrm{m} /$ day, and for sclerotic area 2.07 $(0.72) \mu \mathrm{m} /$ day. There was no significant difference between these values even though the mean values increased progressively from the medial osteoporotic to the lateral sclerotic part of the femoral head.

Osteoclast count, expressed as mean (SD) osteoclast number per $\mathrm{cm}^{2}$, was $0.85(0.95)$ in 
the medial area, $0.97(0.77)$ in the central area, and $1.51(1.07)$ in the lateral area. There was no significant difference between these values.

\section{Discussion}

Remodelling of subchondral bone has been considered to have a key role in the pathogenesis and perpetuation of the changes in osteoarthritis. Bone sclerosis can result from an increased rate of appositional bone formation or decreased resorptive activity, an increased amount of bone forming surface (increased osteoid), and increased enchondral ossification of the calcified layer of the articular cartilage. Ossification also occurs in plugs of metaplastic cartilage within the eburnated bone at the joint surface, but this mechanism probably has a minor role, if any at all, in the alteration of the bony contour of the femoral head.

Bone remodelling has not previously been studied in the subchondral bone of the human osteoarthritic femoral head. Regional differences in density in the osteoarthritic femoral head are well known. This has been shown by bone morphometry in the present study and by Vernon-Roberts (personal communication). The question arises as to whether there is a change in the rate of bone formation or resorption in loaded compared with unloaded parts of the osteoarthritic femoral head. As we have found that the mean appositional growth rate and osteoclast numbers are the same in low and high load bearing areas of the femoral head, some other explanation is required for the differences in bone density. Interestingly, the medial side of the femoral head often shows decreased bone density and may even show the presence of a large cyst-like space due to local osteoporosis, yet this medial part of the femoral head was not inactive with respect to tetracycline incorporation. Appositional bone growth was not different medially, compared with the sclerotic load bearing area, nor was there any significant increase in osteoclastic activity. The difference in density may occur as a result of differences in the total duration of new bone formation or resorption at different sites. It is also possible that there is more bone forming trabecular surface in the areas becoming sclerotic; further studies are required to evaluate the osteoid surface of subchondral bone. The possibility that increased enchondral ossification of the calcified zone of cartilage has a role in the production of a dense subchrondral bone must be considered, but this does not, of course, apply to areas of continuing new bone formation in eburnated regions where no source of enchondral ossification is present apart from small amounts of metaplastic cartilage at the joint surface. Thus enchondral ossification would seem an unlikely mechanism for bone sclerosis in osteoarthritis.

While the appositional bone rate and bone resorptive activity may vary in an individual from time to time, over any particular short period, the rate at which bone may be laid down and resorbed seems to be fairly constant, irrespective of load bearing.

These results are in keeping with our previous findings in relation to rates of bone formation around human autografts and bone allografts. ${ }^{18}$

1 Meachim R. Age changes in articular cartilage. Clin Orthop 1969;64:33-44.

2 Byers PD, Maroudas A, Oztop F, Stockwell RA, Venn MF Histological and biochemical studies on cartilage from osteoarthritic femoral heads with special reference to surface characteristics. Connect Tissue Res 1977;5:41-9.

3 Ali SY, Griffiths S. Formation of calcium phosphate crystals in normal and osteoarthritic cartilage. Ann Rheum Dis 1983;42(suppl 1):45-8.

4 Mankin HJ, Lippiello L. Biochemical and metabolic abnormalities in articular cartilage from osteoarthritic human hips. I Bone foint Surg 1970;52:424-33.

5 Simon SR, Radin EL, Paul IL. The response of joints to impact loading. II. In vivo behaviour of subchondral impact loading. II. In vivo behaviour

6 Radin EL, Paul IL. Does cartilage compliance reduce skeletal impact loads. Arthritis Rheum 1970;13:139-44.

7 Pogrund H, Rutenberg M, Makin M, Robin G, Menczel J, Steinberg R. Osteoarthritis of the hip joint and osteoporosis: a radiological study in a random population sample in Jerusalem. Clin Orthop 1982;164:130-5.

8 Wientroub S, Papo J, Ashkenazi M, Tardiman R, Weissman SL, Salama R. Osteoarthritis of the hip and fracture of the proximal end of the femur. Acta Orthop Scand 1982;53: $261-4$.

9 Dequeker J. The relationship between osteoporosis and osteoarthritis. Clin Rheum Dis 1985;11:271-96.

10 Healey JH, Vigorita VJ, Lane JM. The coexistence and characteristics of osteoarthritis and osteoporosis. $\mathcal{f}$ Bone foint Surg 1985;67:586-92.

11 Foss MVL, Byers PD. Bone density, osteoarthrosis of the hip and fracture of the upper end of the femur. Ann Rheum Dis 1972;31:259-64

12 Roh YS, Dequeker J, Mulier JC. Cortical bone remodelling and bone mass in primary osteoarthritis of the hip. Invest Radiol 1973;8:251-4.

13 Roh YS, Dequeker J, Mulier JC. Bone mass in osteoarthrosis, measured in vivo by photon absorption. $\mathcal{f}$ Bone foint Surg 1974;56:587-91.

14 Roh YS, Dequeker J, Mulier JC. Trabecular pattern of the upper end of the femur in primary osteoarthrosis and in symptomatic osteoporosis. Fournal Belge de Radiologie 1974;57:89-94.

15 Gevers G, Dequeker J, Geusens P, Nyssen-Behets C, Dhem A. Physical and histomorphological characteristics of iliac A. Physical and histomorphological characteristics of iliac crest bone differ according to the
the hand. Bone 1989;10:173-7.

16 Frost HM. Histomorphometry of trabecular bone. I. Theoretical correction of appositional rate measurements. In: Meunier PJ, eds. Bone histomorphometry. 2nd international workshop. Paris: Armour-Montagu, 1976: 362-70.

17 Revell PA. Pathology of bone. New York: Springer Verlag, 1986:94-6.

18 Samuelson KM, Freeman MAR, Levack B, Rassmussen GL, Revell PA. Homograft bone in revision acetabular arthroplasty. $\mathcal{F}$ Bone foint Surg 1988;70:367-72. 